IOSR Journal of Dental and Medical Sciences (IOSR-JDMS)

e-ISSN: 2279-0853, p-ISSN: 2279-0861. Volume 16, Issue 5 Ver. VI (May. 2017), PP 34-35

www.iosrjournals.org

\title{
Comparison of HIV Screening Methods of Donor Blood Using Rapid Fourth Generation Elisa Assay in Kaduna, Nigeria
}

O. U. Daudu ${ }^{1,2}$, M. Ajiboye ${ }^{1}$, S.Ajala ${ }^{1}$, M.Esiekpe ${ }^{1}$ A.S. Buru ${ }^{3}$

${ }^{I}$ National Blood Transfusion Service, Kaduna Nigeria, ${ }^{2}$ FMPC, Gawu-Babangida Niger State Nigeria, ${ }^{3}$ Pathology Department, Faculty Of Medicine, Kaduna State University, Kaduna, Kaduna State, Nigeria

\begin{abstract}
The risks of transmitting HIV infection in blood transfusion can be reduced by improved donor selection and improved method of screening, which is able to reduce the diagnostic window between the time of Human Immunodeficiency Virus (HIV) infection and laboratory diagnosis. Fourth-generation Enzyme-Linked Immunosorbent assays (ELISA) for the combine detection of HIV antigen and antibody were compared to Rapid assays which detect antibody only. A total of 300 negative sera collected from four different hospitals were rescreened using ELISA, out of which 12 of the negative sera tested positive giving prevalence of $4 \% .1(2.77 \%)$ were positive from 36 negative sera, $10(5.8 \%)$ were positive from 171 sera, $1(1.36 \%)$ were positive from 73 negative sera and none was positive from 20 negative sera. The results show that overall HIV combine antigen/antibodies assays present better performance when compared to antibody only assays.
\end{abstract}

Keywords: Elisa Assay, Blood Transfusion, Comparison, Rapid Screening

\section{Introduction}

Blood transfusion is a life-saving intervention. Availability of safe blood and blood products is a critical component in improving health care and in preventing the transmission of infections. Though millions of lives are saved each year through blood transfusion, yet the quality and safety of blood transfusion is of continuing concern, particularly in developing countries where $82 \%$ of the world's population lives. The burden of disease and loss of life resulting from unsafe blood transfusions is devastating for communities in developing countries [1]. Blood transfusion in Nigeria dates back to the 1950s and has remained a challenge to effective health care delivery. Developed countries have come to demand absolute freedom from transfusion-transmitted infection, while simultaneously conceding that zero-risk transfusion is unlikely to ever be achieved [2].

Testing of all donated blood for transfusion transmitted infections (TTIs) such as HIV, Hepatitis B, Hepatitis C and Syphilis is one of the strategies recommended by WHO to ensure safe blood [3,4]. Safe blood, according to WHO is a universal right and blood is referred to as safe if it has been screened for transfusion transmissible infections and is not likely to cause harm to its recipient. The risk of HIV infection through unsafe blood and blood products is exceptionally high (95-100\%) compared to other common routes of HIV exposure. In the year 2000 the World Health Organization, estimated that inadequate blood screening had resulted in 1 million new HIV infections worldwide, which is equivalent to $5 \%$ to $10 \%$ of HIV infections worldwide $[3,5]$. In sub-Saharan Africa, heterosexual is also one of the major routes of HIV transmission [6]. Therefore, the aim of this study was to compare the efficacy of rapid assay (commonly use in many private hospitals, general hospitals including some teaching hospitals) with that of fourth generation ELISA, so that a more reliable, reproducible method of screening blood for HIV will be adopted; thereby shortening of the diagnostic window between infection and the detection of antibodies which significantly reduced occurrence of transmission of HIV through the transfusion of blood and blood products.

\subsection{Scope of Study}

\section{Materials And Methods}

This study was carried out on 300 blood donors who had been screened using rapid assay (Alere Determine HIV-1/2, Trinity Biotech Unigold and Chembio HIV-1/2 Stat Pak Assay as tie breaker) from various hospitals and termed non reactive for HIV, the 300 donor sample were collected from four different hospitals and then screened for HIV using the fourth generation ELISA.

\subsection{Study Area}

The study was carried out within Kaduna metropolis, all negative samples were collected in hospitals within Kaduna. Kaduna is the capital city of Kaduna State located in north-western Nigeria, with the following coordinates $10^{\circ} 20^{\prime} \mathrm{N} 7^{\circ} 45^{\prime} \mathrm{E}$.

\subsection{Method:}

Commercial kits Genscreen ${ }^{\mathrm{TM}}$ ULTRA HIV Ag-Ab -Screen Kit with ELISA Microwell Strips $1 \mathrm{X} 8$ Wells was used for this study, The Genscreen ULTRA HIV Ag-Ab is an enzyme immunoassay based on the principle of the sandwich technique for the detection of HIV antigen and of the various antibodies associated with HIV-1 and/or HIV-2 virus in human serum or plasma. The kits were supplied with all required reagents, as well as, having undergone extensive quality control measures to determine the appropriate working conditions of the assay. Validation of the assay is required by the laboratory prior to clinical use, regardless of the quality control provided by the manufacturer.

III. Results

The results obtained from the 300 samples obtained are shown in the tables 1 and 2 below.

Table 1: Distribution Showing Positive And Negative Results

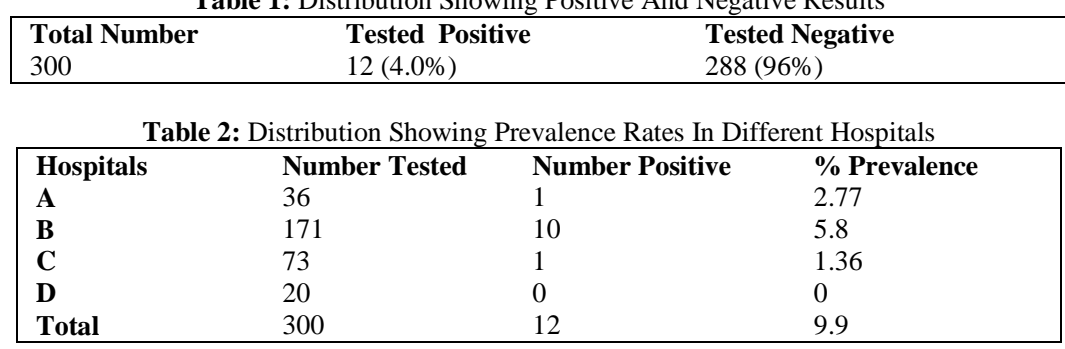




\section{Discussion}

Three hundred (300) rapid assayed negative samples from four hospitals were rescreened using a fourth- generation EnzymeLinked Immunosorbent Assay (ELISA). The results are as shown in Table 1. Twelve (12) samples out of the 300 negative samples previously screened using rapid antibody were found to be positive with fourth generation ELISA method. This agrees with the discovery of [7] and [8] who had 2.2\% positivity (using fourth generation Enzyme Linked Immunsorbent Assay) out of the 87 samples that were formally negative by both rapid assay and third generation ELISA.

The result of this study is in agreement with [8] and tends to suggest that the occurrence of positive samples in previously screened negative samples of rapidly assayed, indicates a possible occurrence of false negative results probably due to the inability of rapid assays to detect antibodies despite the presence of HIV. This would further suggest that antibody testing is unable to detect HIV-infected blood donated by donor in window period that are yet seroconvert $[9,10]$.

The specific prevalence rates for the different hospitals are as shown in Table 2. Hospital A had 2.8\% positivity out of a total 36 negative samples screened, while $5.8 \%$ of samples from hospital B were found to be positive out of a total 171 negative samples screened, and $1.36 \%$ of samples from hospital $\mathrm{C}$ was positive from 73 negative samples screened. There was no positive sample out of 20 negative sample screened from hospital D.

Regardless of the number of samples collected from the four different hospitals used in this study, Hospital B had the highest percentage of positivity from the rescreen negative samples as shown in Table 2. Hospital D had lowest percentage of positivity out of the samples rescreened even though only 20 samples were collected. The false negative result recorded with rapid assays kit in this study could not be limited to a particular rapid assay kit as it was noted that the four hospitals used diverse rapid kit based on availability within the period of this research.

This research also agreed with the study of $[11,12,13]$ whose results all indicated that fourth-generation Enzyme Linked Immunsorbent Assay is able to detect infected sample through the use of antigen testing which test for p24, (viral core).

\section{Conclusion}

The result of this study shows that, the window period is shortened by fourth-generation assays as it permits an earlier diagnosis of HIV infection than third-generation antibody screening assays through the detection of p24 antigen, which may be present in serum samples from individuals with recent HIV infection prior to seroconversion.

For improvement in safety of blood supply, more stringent qualifications such as improvement in donor recruitment, donor education, donor screening, and the use of blood screening assay that can detect infection during the window period should be encouraged.

\subsection{Suggestion and Recommendation}

The residual risk of HIV transmission by blood as indicated in this work is evidence that the safety of donated blood could be improved by the use of assay that combines testing for both antibody and antigen.

In view of HIV/AIDS pandemic and the need to achieve total safety in blood transfusion, the development and implementation of a road map for blood safety can ultimately result in a safe, high quality blood supply for all patients requiring blood transfusion and the elimination of unsafe transfusion as a route of HIV transmission.

\section{Strategy for blood safety and availability:}

1. Collection of blood from voluntary non-remunerated blood donors at low risk of infections that can be transmitted through blood and blood products, the phasing out of family/replacement donation and the elimination of paid donation.

2. Rational use of blood to reduce unnecessary transfusions and minimize the risks associated with transfusion, the use of alternatives to transfusion, where possible, and safe clinical transfusion procedures.

3. Implementation of effective quality systems, including quality management, the development and implementation of quality standards, effective documentation systems, training of all staff and regular quality assessment.

4. Screening of all blood donations should be mandatory using a combination of HIV antigen-antibody assay.

\section{References}

[1]. Dhingra N, Lloyd SE, Fordham J, Amin, NA. Challenges in global blood safety. World Hospitals Health Serv. 2004; 40(1):45-9, 51, 52.

[2]. Klein, HG. Will Blood Transfusion Ever Be Safe Enough? Journal of the American Medical Association; 2000; 284:238-240

[3]. Improving blood safety worldwide. Lancet editorial 2007; 370 www.thelancet.com

[4]. WHO 2010. Screening Donated Blood for Transfusion-Transmissible Infections. www.who.int/diagnostics_laboratory/publications/evaluations/ en/index.html

[5]. UNAID 1997. Impact of HIV and Sexual Health Education on the Sexual Behaviour of Young People: A Review Update. data.unaids.org/publications/IRC-pub01/jc010-impactyoungpeople_en.pdf

[6]. WHO 2002. Safe Blood and Blood Products. Module 2: Screening for HIV and Other Infectious Agents. www.who.int diagnostics_laboratory/ evaluations.

[7]. Odunukwe NN, Njoku SO, Nnodu O, Amusu AO. Risk of HIV transmission through "SCREENED" blood transfusion in Nigeria; International Conference on AIDS, 2004 Jul 11-16 ( Bangkok, Thailand). 15: abstract no. C10092.

[8]. Preena B, Sargan K, Sanjim C, Hitender G. Early Diagnosis of Primary HIV Infection by Fourth Generation ELISA in Spouses of HIV-Positive Patients and STD Clinic Attendees Journal of the International Association of Physicians in AIDS Care 2011; 1 vol. 10 no. 2 69-70.

[9]. Hare CB, Pappalardo BL, Busch MP, Phelps B, Alexander SS, Ramstead C, Levy JA, Hecht FM. "Negative HIV antibody test results among individuals treated with antiretroviral therapy (ART) during acute/early infection". The XV International AIDS Conference. pp. 2004. Abstract no. MoPeB3107.

[10]. CDC 2007. HIV Testing Basics for Consumers, from the Seattle and King County Public Health Department. http://www.cdc.gov/ hiv/topics/testing/resources/qa/index.htm.

[11]. Weber B, Fall E H M, Berger A, Doerr H W. Reduction of diagnostic window by new fourth-generation human immunodeficiency virus screening assays. J Clin Microbiol. 1998; 36:2235-2239

[12]. Laperche S, Maniez-Montreuil M, Couroucé AM. Screening tests combined with p24 antigen and anti-HIV antibodies in early detection of HIV-1. Transfusion Clinique et Biologique, Suppl. 2000; 1:18s-24s.

[13]. Ly TD, Laperche S, Brennan C, Vallari A, Ebel A, Hunt J, Martin L, Daghfal D, Schochetman G, Devare S. Evaluation of the sensitivity and specificity of six HIV combined p24 antigen and antibody assays. Journal of Virological Methods. 2004; 122(2):185-94 